\title{
Actual Chronic Disease Treatment Effects with Drug Treatment Behavior Options: Evidence from China Health and Retirement Longitudinal Study Survey
}

\author{
Minya Li* \\ Department of Psychology, Zhongnan University of Economics \& Law, China
}

*Corresponding author: Minya Li, Department of Psychology school of Finance, Zhongnan University of Economics \& Law, Wuhan 430073, China

\begin{abstract}
The study aims to examine the actual chronic disease treatment effect and drug treatment behaviour options in China through an empirical analysis of associated theories. Using the data from China Health and Retirement Longitudinal Study (CHARLS 2013) collated by Peking University's National Institute of Development, the actual chronic disease treatment effects with drug treatment behaviour options were tested. An analysis was conducted on 13 common chronic diseases. To test the hypothesis, Chinese residents' health condition distribution for both 'chronic disease' and 'all observations' groups were developed and descriptively analysed. It was found that chronic diseases can have an apparent impact on one's perception of physical health. Moreover, by segmenting the different drug therapy behaviours for 13 common types of chronic diseases, it was found that such behaviours were quite varied; for example, for most people with chronic kidney disease, they have opted not to treat it. On this basis, the treatment effects with different therapeutic behaviour selections for these chronic diseases were further explored. This study has provided several contributions. First, it was validated that chronic diseases have a significant negative effect on people's perception of their own health. Second, the list of dominating chronic conditions and their combinations can present a pragmatic start for the development of necessary guidelines related to multimorbidity. Third, a number of new insights have arisen with regard to approach selections for treatment of chronic diseases.
\end{abstract}

Keywords: Multimorbidity; Herapeutic behaviour selections; Medical insurance

\section{Introduction}

It is obvious that most drugs have pros and cons even though many attempts towards safer and predictable treatment effects [1]. However, it is still a lot of academic gaps in this area, as such comprehensive data actually hard to available, especially relate to the different drug treatments effect. Further epidemiological studies of actual treatment effect occurrence are needed to find out possible proper approaches when choosing what drug to treatment options [2], and this paper is try to evaluate the different methods implemented to cure chronic diseases, and we choose 13 types common chronic diseases, namely, hypertension, dyslipidemia (include elevation of low density lipoprotein, triglycerides (TGs), and total cholesterol, or a low high density lipoprotein level), diabetes or high blood sugar, cancer or malignant tumor, Chronic lung diseases, such as chronic bronchitis, emphysema, liver disease (except fatty liver, tumors, and cancer), heart attack, coronary heart disease, angina, congestive heart failure, or other heart problems, stroke, kidney disease (except for tumor or cancer), stomach or other digestive disease (except for tumor or cancer), emotional, nervous, or psychiatric problems, memory-related disease, arthritis or rheumatism.

Blood biomarkers provide critical information about the health of older populations, however, due to lack of access to health care [3]. It is not easy to collect blood samples in representative population surveys in such big countries like China. CHARLS survey, more than 13000 respondents located in 150 counties around China donated whole blood, and respondents also answered the question related to high blood pressure, on this basis, our research collected the previous and calculated chronic disease treatment effects with drug Treatment behavior options, and we got other 12 chronic diseases data and treatment data in the similar way. As the largest developing country, China's medical expenses have been 
rising, bringing a huge financial burden to patients [4]. To avoid the high cost of health care, many Chinese people, especially the uninsured ones, choose self-medication as their first option when they encounter a need for health care-even for serious infectious diseases. Therefore, participating medical insurance, no matter what kind of medical insurance, is of vital importance for Chinese residents, especially for those in middle age and old age [5]. On this basis, in our research we also put whether participant medical insurance as one of the key variables into analysis.

Most of existing literature regard to the above mentioned common chronic diseases tend to focus on one drug and its treatment effect, or the function mechanism. Kwon, Oh et al. examined that sterile pyuria occurs in CKD because of chronic renal parenchymal inflammation, and they found that the degree of pyuria, percentage of neutrophils, and presence of urinary nitrites remained independently associated with sterile pyuria [6]. In addition, Whitlock et al made a safety comparison of metformin vs sulfonylurea Initiation in patients with type 2 diabetes and Chronic kidney disease [7]. Houghton (2018) explored the common mechanisms for chronic obstructive pulmonary disease (COPD) and lung cancer [8]. Weiss (2018) analyzed cell-based therapy for chronic obstructive pulmonary disease [9]. Eugenio (2019) researched morbidity and mortality in a population of patients affected by heart failure and chronic obstructive pulmonary disease [10]. Roberto (2015) explored cognitive dysfunction in severe chronic obstructive pulmonary disease (COPD) with or without Long-Term Oxygen Therapy [11]. There are many other studies on chronic diseases, besides, many researches also try to examine different chronic diseases and how they link to each other, and increasing more literature focus on one chronic disease and try to analysis the treatment effect [12-15] In 2018, the statistical information center of the national health and family planning commission released the national health service survey and analysis report [16], which showed that the proportion of elderly people aged 60 and above suffering from two or more chronic diseases was $16.2 \%$. Multimorbidity is a common problem in the elderly and its occurrence rises with age [17]. The co-existence and co-use of chronic diseases increase the possibility of potential drug interactions. A prospective study involving 18,820 patients [18] found that when patients were followed for more than six months, the readmission rate was $6.5 \%$ due to drug interactions, which increased substantial medical expenditures. A systematic review further demonstrated that among older Chinese adults (age more than 60 years), the overall prevalence of multimorbidity was up to $87.0 \%$ in urban residents. However, very little information is available regarding the prevalence of multimorbidity in rural China. Therefore, it is necessary implement a population-based study on chronic diseases treatment and the actual effect evaluation, and it is logical for further development to set up a monitoring system that seeks regularly to document treatment outcomes and types of medication used by patients with chronic infectious and noninfectious diseases, like what Cambodia and Ethiopia did [19].

\section{Research Design \\ Data source and sample selection}

The data for this article comes from the 2013 China Health and Retirement Longitudinal Study, or CHARLS, a large nationwide sampling project led by Peking University's national institute of development that collects high-quality, microscopic data of families and individuals in China [20]. The access response rate and data quality of CHARLS are among the highest among similar projects in the world, and the data has been widely used and recognized in academia. In 2013, CHARLS conducted the second survey, which included basic personal information, family structure and financial support, health status, physical measurement, medical service utilization and health insurance, work, retirement and pension, income, consumption, assets, and basic community information. The data cover personal characteristics, chronic conditions and different drug treatment behavior options. It is the representative large sample open database available at present. In this study, respondents who met the following characteristics were selected as samples: respondents who had made a clear response to their age but if age below 45 years old, then excluded. In order to avoid abnormal values influence on the result, because the revenue collected from some questionnaires, interclass difference is too big, in order to overcome the influence of extreme value, on the part of low income and high income shrink tail processing, in addition to the $0.01 \%$ of extreme high income percentile and $0.01 \%$ of the extreme low percentile data, in addition, we conducted logarithmic processing on the income data, and the number of samples used for regression analysis in this paper was 18029.

\section{Variables measurement}

Explained variable. In this research, the explained variable is the actual effect of treatment on people older than 45 with chronic disease. Based on questionnaire code DA001 and DA002 to healthy problem, in order to guarantee the consistency of the direction of the research question to measure our assignment for measuring the five-point scale to reset, the higher the score shows better health, and then choose proper option according to their own situation(see the variable health). The groups we considered were mainly aimed at people with fair and poor health. On the basis of the questionnaire, we reconstructed the variable subhealth, when the variable health equals to 1-4. As our research target population were treated with different types of drugs, so the variable subhealth could measure their therapeutic effect. Core explanatory variable. Our core explanatory variables include different types of chronic diseases and different drug treatment behaviors. In our study, we selected 13 types common chronic diseases, and the variables in the paper are ch1-ch13 respectively, representing hypertension (variable ch1), dyslipidemia (variable ch2, include elevation of low density lipoprotein, triglycerides (TGs), and total cholesterol, or a low high density lipoprotein level), diabetes or high blood sugar (variable ch3), cancer or malignant tumor (variable ch4), Chronic lung diseases, such as chronic bronchitis, emphysema (variable 
ch5), liver disease (except fatty liver, tumors, and cancer, see variable ch6), heart attack, coronary heart disease, angina, congestive heart failure, or other heart problems (variable ch7), stroke (variable ch8), kidney disease (except for tumor or cancer, see variable ch9), stomach or other digestive disease (except for tumor or cancer, see variable ch10), emotional, nervous, or psychiatric problems (variable ch11), memory-related disease (variable ch12), arthritis or rheumatism (variable ch13). And the different drug treatment options are shown as Tables $1 \& 2$. In addition, as the drug treatment behavior options in the questionnaire for $\operatorname{ch} 2, \operatorname{ch} 5, \operatorname{ch} 6, \operatorname{ch} 7, \operatorname{ch} 9$, ch10, ch12 and ch13 are consistent, i.e. take Chinese traditional medicine, take modern medicine, and other treatments, in this study for clarity we put them together as (Table 3). Meanwhile, as ch1, ch3, ch4, ch8 and ch11 have different treatment behavior options, so that we investigated them separately due to their options, as detailed in Table4.

Table 1: Chinese residents' health condition distribution (\%).

\begin{tabular}{|c|c|c|c|}
\hline Health level & All Observations & Chronic=1 & Chronic=0 \\
\hline Poor & 10.98 & 16.24 & 9.00 \\
\hline Not good & 36.32 & 39.29 & 35.20 \\
\hline Uncomfortable occasionally & 34.58 & 33.04 & 35.17 \\
\hline Fair & 12.74 & 8.52 & 14.33 \\
\hline Good & 5.39 & 2.91 & 6.31 \\
\hline
\end{tabular}

Table 2: Descriptive statistics of main control variables.

\begin{tabular}{|c|c|c|c|c|c|c|c|}
\hline Variable & $\mathbf{N}$ & Mean & Sd & Min & Max & Skewness & Kurtosis \\
\hline health & 16489 & 2.650 & 1.010 & 1 & 5 & 0.410 & 2.810 \\
\hline medins & 17934 & 0.960 & 0.200 & 0 & 1 & -4.650 & 22.61 \\
\hline age & 18029 & 60.27 & 10 & 45 & 102 & 0.590 & 2.770 \\
\hline gender & 18026 & 0.480 & 0.500 & 0 & 1 & 0.0700 & 1 \\
\hline account & 17985 & 0.230 & 0.420 & 0 & 1 & 1.300 & 2.690 \\
\hline edu2 & 18019 & 1.390 & 0.580 & 1 & 4 & 1.220 & 3.580 \\
\hline marry & 18018 & 2.290 & 0.670 & 1 & 4 & 1.860 & 5.110 \\
\hline spouse & 18018 & 0.870 & 0.340 & 0 & 1 & -2.150 & 5.610 \\
\hline area & 18037 & 0.400 & 0.490 & 0 & 1 & 0.410 & 1.170 \\
\hline district & 18037 & 0.310 & 0.460 & 0 & 1 & 0.810 & 1.650 \\
\hline area distr & 18037 & 2.110 & 1.100 & 1 & 4 & 0.380 & 1.720 \\
\hline perincome & 911 & 9.290 & 1.350 & 4.610 & 12.72 & -0.440 & 2.260 \\
\hline
\end{tabular}

Table 3: Treatment options for chronic disease types ch2, ch5, ch6, ch7, ch9, ch10, ch12, ch13.

\begin{tabular}{|c|c|c|c|c|c|}
\hline & Freq. & Percent & & Freq. & Percent \\
\hline \multicolumn{3}{|c|}{ Ch2 } & \multicolumn{3}{|c|}{ Ch5 } \\
\hline Take Chinese traditional medicine & 63 & 4.68 & Take Chinese traditional medicine & 80 & 5.71 \\
\hline Take modern medicine & 588 & 43.72 & Take modern medicine & 641 & 45.79 \\
\hline Other treatments & 92 & 6.84 & Other treatments & 94 & 6.71 \\
\hline \multicolumn{3}{|c|}{ Ch6 } & \multicolumn{3}{|c|}{ Ch7 } \\
\hline Take Chinese traditional medicine & 19 & 5.19 & Take Chinese traditional medicine & 158 & 9.16 \\
\hline Take modern medicine & 126 & 34.43 & Take modern medicine & 912 & 52.87 \\
\hline Other treatments & 14 & 3.83 & Other treatments & 89 & 5.16 \\
\hline \multicolumn{3}{|c|}{ Ch9 } & \multicolumn{3}{|c|}{ Ch10 } \\
\hline Take Chinese traditional medicine & 83 & 10.99 & Take Chinese traditional medicine & 211 & 6.38 \\
\hline Take modern medicine & 216 & 28.61 & Take modern medicine & 1639 & 49.53 \\
\hline Other treatments & 64 & 8.48 & Other treatments & 140 & 4.23 \\
\hline \multicolumn{3}{|c|}{ Ch12 } & \multicolumn{3}{|c|}{ Ch13 } \\
\hline Take Chinese traditional medicine & 9 & 5.49 & Take Chinese traditional medicine & 323 & 6.73 \\
\hline Take modern medicine & 61 & 37.20 & Take modern medicine & 1404 & 29.27 \\
\hline Other treatments & 10 & 6.10 & Other treatments & 1094 & 22.81 \\
\hline
\end{tabular}


Table 4: Treatment options for chronic disease types ch1, ch3, ch4, ch8, ch11.

\begin{tabular}{|c|c|c|c|c|c|}
\hline & Freq. & Percent & & Freq. & Percent \\
\hline \multicolumn{3}{|c|}{ Ch1 } & \multicolumn{3}{|c|}{ Ch3 } \\
\hline Take Chinese traditional medicine & 188 & 4.87 & Take Chinese traditional medicine & 49 & 5.65 \\
\hline Take modern medicine & 2914 & 75.53 & Take modern medicine & 464 & 53.52 \\
\hline- & - & - & Take insulin injections & 163 & 18.80 \\
\hline \multicolumn{3}{|c|}{ Ch4 } & \multicolumn{3}{|c|}{ Ch8 } \\
\hline Take Chinese traditional medicine & 4 & 3.36 & Take Chinese traditional medicine & 17 & 5.88 \\
\hline Take modern medicine & 23 & 19.33 & Take modern medicine & 104 & 35.99 \\
\hline Chemotherapy & 7 & 5.88 & Physical therapy & 6 & 2.08 \\
\hline Surgery & 33 & 27.73 & Acupuncture and moxibustion & 25 & 8.65 \\
\hline Radiation therapy & 11 & 9.24 & Occupational therapy & 3 & 1.04 \\
\hline \multicolumn{6}{|c|}{ Ch11 } \\
\hline Psychological treatment & 10 & 6.71 & & & \\
\hline Take anti-depressants & 11 & 7.38 & & & \\
\hline Take tranquilizers or sleeping pills & 41 & 27.52 & & & \\
\hline
\end{tabular}

Other explanatory variables and control variables. Based on the CHARLS2013 questionnaire and the analysis of influencing factors of treatment effect in the existing literature, a series of control variables were selected in this paper. Among them, personal characteristics mainly include age, gender, education level, income, residential area, marital status, etc. At the same time, since the questionnaire did not directly ask the age of the respondents, but marked with "year of birth", this paper adopted the method of subtracting the year of birth of the respondents from 2013 to convert it into a continuous variable. The square of age was also included in the study, taking into account the possible non-linear relationship between the actual therapeutic effect of different drugs and chronic diseases.

\section{Model design}

Due to the discrete variables of actual drug treatment effect, the discrete dependent variable probabilistic model (Logit or Probit) is often used in the existing studies for parameter estimation. At the same time, according to the findings, whether OLS model is used for linear regression or discrete variable model, the direction and significance of parameter estimation are consistent, and the influence on coefficient value will not change too much. Therefore, ordinary least square method (OLS) is not used to estimate model parameters [21-24]. However, the regression results of the normal discrete dependent variable probabilistic model can only draw the fuzzy conclusion of positive effect and negative effect but cannot see the specific effect of each unit change on the explained variable. As the purpose of this study is to investigate the effect of specific drug treatment behavior on the actual quality effect, on this base, we analyzed the marginal effect, and the empirical results of this study are also about the output of specific marginal effect. The specific form of the empirical model is as follows:

Treatment effecti $=\alpha 0+\alpha 1$ Treatmentoptionsi $+\alpha 2$ medinsi $+\alpha 3 \mathrm{Xi}$

In the model, Treatment effect represents the quality effect of the ' $i$ ' survey object, medins is the dummy variable of medical insurance, considering that in China, whether residents participate in medical insurance has a great influence on how they treat their diseases, so whether they participate in medical insurance is an important variable. Xi represents the control variable vector group, including age and its quadratic terms, education level, household registration, gender, etc., as shown in Table 2 . In the empirical research, the general probit and logit regression focuses on the positive and negative direction and significance of the coefficient, rather than the value of the coefficient. For the convenience of research, we recalculated the marginal ratio based on the preliminary regression results, so the coefficients of the results later in this paper are statistically significant. All the empirical analysis was calculated by Stata15.0.

\section{Empirical Results and Discussion \\ Descriptive analysis}

Table 1 below shows the distribution of chronic diseases under different health conditions. As mentioned, the health level data was collected according to respondents' answers of question DA001 and DA002, and we collected 16489 effective data of health conditions. And we can see that the answer to this question involves poor, not good, uncomfortable occasionally, fair and good. In the whole sample, $36.32 \%$ and $34.58 \%$ of the respondents felt that their physical health was average. Our survey subjects are Chinese residents over the age of 45 . Although most people think they are in average health, this result is consistent with the reality considering their older age. In addition, for all observations, 10.98 percent of people thought they were in poor health, while only 5.39 percent thought they were in good health. CHARLS data is a representative database with 16,489 samples. The distribution of physical health status largely represents the physical health status of Chinese residents.

(Table 1) also explores the prevalence of chronic diseases and their health conditions distribution. There are 13 common chronic diseases that we research. Here, we only examine whether people had chronic diseases. That is to say, as long as the answer includes 
at least one chronic disease, then we consider it classified into the group with chronic disease $=1$. Then, let's look at the health distribution of people with chronic diseases. The group with chronic disease had worse overall health than the entire sample. As we can see, the proportion of respondents with very poor and poor health status is more than the proportion of the whole sample, while only $2.91 \%$ of them think their health status is good. This reflects a lack of consideration for the severity of chronic diseases, but they do have a strong influence on how people perceive their health. For the group without chronic disease, their health was slightly better than for the full sample. Although there are as many as 13 chronic diseases in our study, most people still do not suffer from chronic diseases. Therefore, overall, the data in (Table 1) is robust. In our research, we controlled some variables that may affect our empirical regression result. Table 2 is descriptive statistics for control variables. The maximum age is 102 , and the average age is 60 . In the following empirical analysis, we actually added the square of age into regression model because previous literature suggested that there might be an inverted U-shaped relationship between age and the explained variable. As for the gender variable, we set gender $=1$ if answer male, and 0 if female. This suggests that women are slightly more likely to be older than men in the elderly group, which is a preliminary reflection of the fact that women may be older than men. The variable account means whether it is an agricultural or non-agricultural, although the account conditions for residents in China not important as past, we still include this variable in our analysis because our survey subjects are people over the age of 45 , for whom migration in China may have little impact. Variable edu is calculated by education level, according to CHARLS questionnaire, the level of education divided into a lot of classes, we set score equals to 1 if the primary education level, 2 if junior or high school level, 3 if attended universities, and 4 if graduate. In addition, the variable area means residence cities or the countryside, and district is located in the coastal areas or inland, readier involves 1 if rural inland, 2 if coastal rural area, 3 if inland cities, and 4 if coastal cities. Besides, the skewness and kurtosis of the variables shows that the data is very smooth, and fairly evenly distributed (Table 2).

For the group with chronic diseases, different people have different drug treatment behavior choices. In the CHARLS questionnaire, there are three medication behavior options for ch2, ch5, ch6, ch7, ch9, ch10, ch12, ch13. In order to make it clear, we categorize these as Table 3. The treatment behaviors for these chronic diseases are: taking traditional Chinese medicine, taking western medicine and other treatments. We can see that in fact, the number of people suffering from various chronic diseases is not very large. However, according to the distribution of people's health status in Table 1, chronic diseases greatly affect people's perception of health, and these people are more pessimistic. From the frequency of Table 3, the number of patients with Ch10 and Ch13 are the largest, and western medicine is the mainstream treatment for all patients with chronic diseases. For some people with chronic diseases, especially Ch9 kidney disease, most people with this chronic disease choose not to seek treatment (Table 3).

Table 4 is treatment options for chronic disease types ch1, ch3, ch4, ch8, ch11.People with high blood pressure, chronic Ch1 disease and Ch3 diabetes would choose treatment. However, only a few people know that they are suffering from mental illness, and most people will not take measures to treat mental illness, which shows that residents' understanding of mental illness is not broad enough in China. Surprisingly, most people with mental illness choose sedatives or sleeping pills over professional treatments and antidepressants. Overall, the proportion of taking western medicine is the mainstream of treatment for these chronic diseases (Table 4).

\section{Treatment effects with different therapeutic behavior selections regression analysis}

For different types of chronic diseases, some of the actual quality effects are effective, while others cannot be concluded to improve physical health regardless of the drug treatment. (Tables 5\&6) are the regression results of the actual quality effects and their drug treatment behavior choices. First, with respect to $\mathrm{Ch} 2$ dyslipidemia and Ch10 stomach or other digestive disease, both traditional Chinese medicine treatment and western medicine treatment can effectively and significantly improve health. As for chronic Ch5 lung disease, taking traditional Chinese medicine and other treatments improves health, while taking western medicine does not. For Ch7 heart disease, Ch8 stroke, taking western medicine is the most effective treatment effect to improve physical health (Table 5).

Table 5: Treatment effects with different therapeutic behavior selections for ch2, ch5, ch6, ch7, ch9, ch10, ch12 and ch13 (Marginal Effect).

\begin{tabular}{|c|c|c|c|c|c|c|c|c|}
\hline & $\mathbf{( 1 )}$ & $\mathbf{( 2 )}$ & $\mathbf{( 3 )}$ & $\mathbf{( 4 )}$ & $\mathbf{( 5 )}$ & $\mathbf{( 6 )}$ & $\mathbf{( 7 )}$ & $\mathbf{( 8 )}$ \\
\hline $\begin{array}{c}\text { Take Chinese } \\
\text { traditional } \\
\text { medicine }\end{array}$ & $0.091^{* * *}$ & $0.047^{* *}$ & -0.082 & 0.023 & 0.030 & $0.051^{* * *}$ & 0.010 & 0.019 \\
\hline $\begin{array}{c}\text { Take modern } \\
\text { medicine }\end{array}$ & $0.066^{* * *}$ & 0.002 & 0.015 & $0.051^{* * *}$ & 0.024 & $0.049^{* * *}$ & 0.053 & $0.041^{* * *}$ \\
\hline $\begin{array}{c}\text { Other } \\
\text { treatments }\end{array}$ & 0.019 & $0.052^{* * *}$ & -0.008 & 0.007 & -0.005 & $0.073^{* * *}$ & -0.019 & $0.030^{* * *}$ \\
\hline $\begin{array}{c}\text { Other control } \\
\text { variables }\end{array}$ & $\sqrt{ }$ & $\sqrt{ }$ & $\sqrt{ }$ & $\sqrt{ }$ & $\sqrt{ }$ & $\sqrt{ }$ & $\sqrt{ }$ & $\sqrt{ }$ \\
\hline $\mathrm{N}$ & 1989 & 1984 & 554 & 2228 & 1112 & 4190 & 274 & 5894 \\
\hline pseudo R2 & 0.035 & 0.017 & 0.054 & 0.036 & 0.034 & 0.033 & 0.088 & 0.013 \\
\hline
\end{tabular}


However, at the same time, we see Ch6, Ch9, Ch12, and Ch4, Table 6's Ch11, CHARLS data results reflect the treatment of chronic diseases, can significantly improve the health level, the cause of this result may be that on this several chronic diseases, CHARLS questionnaire design can't cover the people real situation, cause the sample selection bias, it is possible that the sample size is too small, unable to draw valid estimates. For chronic Ch1 hypertension treatment, the treatment of western medicine effect effectively, and the traditional Chinese medicine is not effective in relieving the condition of high blood pressure, although this result is surprising, but we see in Table 4, choose with traditional Chinese medicine in the treatment of high blood pressure, effective samples is 188 , maybe the sample is too small for the actual treatment effect. For chronic Ch3diabetes or high blood sugar, although some people choose to inject insulin to treat $\mathrm{Ch} 3$, our CHARLS regression conclusion is that taking western medicine can effectively control the disease. Although insulin seems to improve significantly, more samples are needed for further testing (Table 6).

Table 6: Treatment effects with different therapeutic behavior selections for ch1, ch3, ch4, ch8 and ch11 (Marginal Effect).

\begin{tabular}{|c|c|c|c|c|c|}
\hline & (1) & (2) & (3) & (4) & (5) \\
\hline & Ch1 & Ch3 & Ch4 & Ch8 & Ch11 \\
\hline $\begin{array}{c}\text { Take Chinese } \\
\text { traditional medicine }\end{array}$ & 0.022 & 0.284 & -0.051 & -0.269 & \\
\hline Take modern medicine & $0.061^{* * *}$ & $0.240^{* * *}$ & -0.006 & 0.096 & \\
\hline Take insulin injections & & 0.280 & & & \\
\hline Chemotherapy & & & 0.038 & & \\
\hline Surgery & & & 0.027 & & \\
\hline Radiation therapy & & & - & & \\
\hline Physical therapy & & & & -0.237 & \\
\hline $\begin{array}{l}\text { Acupuncture and } \\
\text { moxibustion }\end{array}$ & & & & $0.108^{*}$ & \\
\hline Occupational therapy & & & & -0.105 & \\
\hline $\begin{array}{c}\text { Psychological } \\
\text { treatment }\end{array}$ & & & & & - \\
\hline Take anti-depressants & & & & & -0.265 \\
\hline $\begin{array}{l}\text { Take tranquilizers or } \\
\text { sleeping pills }\end{array}$ & & & & & -0.119 \\
\hline Other control variables & $\sqrt{ }$ & $\sqrt{ }$ & $\sqrt{ }$ & $\sqrt{ }$ & $\sqrt{ }$ \\
\hline $\mathrm{N}$ & 4663 & 1215 & 158 & 428 & 195 \\
\hline pseudo R2 & 0.020 & 0.030 & 0.091 & 0.059 & 0.081 \\
\hline
\end{tabular}

\section{Conclusion}

Across our study, firstly we found chronic diseases have a significant negative effect on people's perception of their own health. Then by segmenting treatment options for 13 types chronic disease, considering that the main research objectives are Chinese residents aged above 45 years old, in real life experience, traditional Chinese medicine treatment is one of a common options for this group to some extent, therefore in our study, we still take the treatment option as take Chinese tradition medicine into consideration. We also found evidence for the actual chronic disease treatment effect varies a lot by different drug treatment behavior options. Among the common 13 types chronic disease, taking modern medicine is the mainstream treatment choice and most time it is effective. The current study also shows that for chronic diseases ch2 dyslipidemia, ch5 chronic lung diseases and ch10 chronic stomach or other digestive disease, take Chinese traditional medicine are effective. And for ch8 stroke, acupuncture and moxibustion are effective treatment approaches. The implications derived from this study can help provide a synthetical analysis for us to explore the treatment effect under different medication behavior options for different kind of chronic diseases, thus enabling a better evaluation of chronic diseases treatments and choose a proper medication approach for these chronic diseases.

\section{References}

1. Alvarez PA, Bril F, Castro V, Meiville I, Gonzalez CD, et al. (2013) Adverse drug reactions as a reason for admission to aninternal medicine ward in Argentina. Int J Risk Saf Med 25(3): 185-192.

2. Boyd CM, Darer J, Boult C, Fried LP, Boult L, et al. (2005) Clinical practice guidelines and quality of care for older patients with multiple comorbid diseases. JAMA 294(6): 716-724.

3. Eugenio RC, Matteo L, Crescenzio B, Spinardi L, Esposti DD, et al. (2019) Morbidity and mortality in a population of patients affected by heart failure and chronic obstructive pulmonary disease: an observational study. BMC Cardiovasc Disord 19(1): 20.

4. Carbonell FA, Frijers P (2004) How important is methodology for the estimates of the determinants of Happiness? The Economic Journal 114(497): 641-659.

5. France EF, Wyke S, Gunn JM, Mair FS, McLean G, et al. (2012) Multimorbidity in primary care: a systematic review of prospective cohort studies. Br J Gen Pract 62(597): e297-e307.

6. Houghton AM (2018) Common mechanisms linking chronic obstructive pulmonary disease and lung cancer. Ann Am Thorac Soc 15(Suppl 4): S273-S277. 
7. Janssens B, Van Damme W, Raleigh B, Gupta J, Khem S, et al. (2007) Offering integrated care for HIV/AIDS, diabetes and hypertension within chronic disease clinics in Cambodia. Bull World Health Organ 85(11): 880-885.

8. Patinen JL, Neuvonen PJ (2002) Drug-related deaths in a university central hospital. Eur J Clin Pharmacol 58(7): 479-482.

9. Kwon YE, Oh DJ, Kim MJ, Choi HM (2020) Prevalence and clinical characteristics of asymptomatic pyuria in chronic kidney disease. Ann Lab Med 40(3): 238-244.

10. Mamo Y, Seid E, Adams S, Gardiner A, Parry E (2007) A primary healthcare approach to the management of chronic disease in Ethiopia: an example for other countries. Clin Med 7(3): 228-231.

11. Marengoni A, Rizzuto D, Wang HX, Winblad B, Fratiglioni L (2009) Patterns of chronic multimorbidity in the elderly population. J Am Geriatr Soc 57(2): 225-230.

12. Nagamatsu A, Kawaguchi T, Hirota K, Koya S, Tomita M, et al. (2019) Slow walking speed overlapped with low handgrip strength in chronic liver disease patients with hepatocellular carcinoma. Hepatol Res 49(12): $1427-1440$

13. Neuvonen PJ (2012) Towards safer and more predictable drug treatment-reflections from studies of the first BCPT prize awardee. Basic Clin Pharmacol Toxicol 110(3): 207-218.

14. Ooms G, Van Damme W, Baker BK, Zeitz P, Schrecker T (2008) The "diagonal" approach to Global Fund financing: a cure forthe broader malaise of health systems? Global Health 4: 6 .

15. Peng YC, Chang WH, Zhou HQ Hu HP, Liang WN (2010) Factors associated with health-seeking behavior among migrant workers in Beijing, China. BMC Health Serv Res 10: 69.

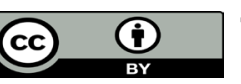

This work is licensed under Creative

Commons Attribution 4.0 License

To Submit Your Article Click Here:

Submit Article
16. Pirmohamed M, James S, Meakin S, Green C, Scott AK, et al. (2004) Adverse drug reactions as cause of admission to hospital: prospective analysis of 18820 patients. BMJ 329(7456): 15-19.

17. Pitychoutis PM, Zisaki A, Dalla C, Daifoti ZP (2010) Pharmacogenetic insights into depression and antidepressant response: does sex matter? Curr Pharm Des 16(20): 2214-2223.

18. Roberto WN, Luca B, Fernanda PB, Tognella S, Turco P (2015) Cognitive dysfunction in severe chronic obstructive pulmonary disease (COPD) with or without Long-Term Oxygen Therapy (LTOT). Multidiscip Respir Med 10(1): 17.

19. Robinson JA, Sareen J, Cox BJ, Bolton JM (2009) Correlates of self-medication for anxiety disorders: results from the National Epidemiologic Survey on Alcohol and Related Conditions. J Nerv Ment Dis 197(12): 873-878.

20. WHO (2018) Statistical information center of the national health and family planning commission. National health service survey and analysis report, WHO, Switzerland.

21. Stewart DE, Ashraf IJ, Munce SE (2006) Women's mental health: a silent cause of mortality and morbidity. Int J Gynaecol Obstet 94(3): 343-349.

22. Weiss DJ (2018) Cell-based therapy for chronic obstructive pulmonary disease. Rebuilding the lung. Ann Am Thorac Soc 15(Suppl 4): S253-S259.

23. Whitlock RH, Hougen I, Komenda P, Rigatto C, Clemens KK, et al. (2020) A safety comparison of metformin vs sulfonylurea initiation in patients with type 2 diabetes and chronic kidney disease: a retrospective cohort study. Mayo Clin Proc 95(1): 90-100.

24. Xiayan D, Linhong C, Zhiming X, Xiaocang X (2019) An assessment of the economic burden of senile chronic diseases in China based on China Health and Retirement Longitudinal Survey. Expert Rev Pharmacoecon Outcomes Res 5: 1-8.

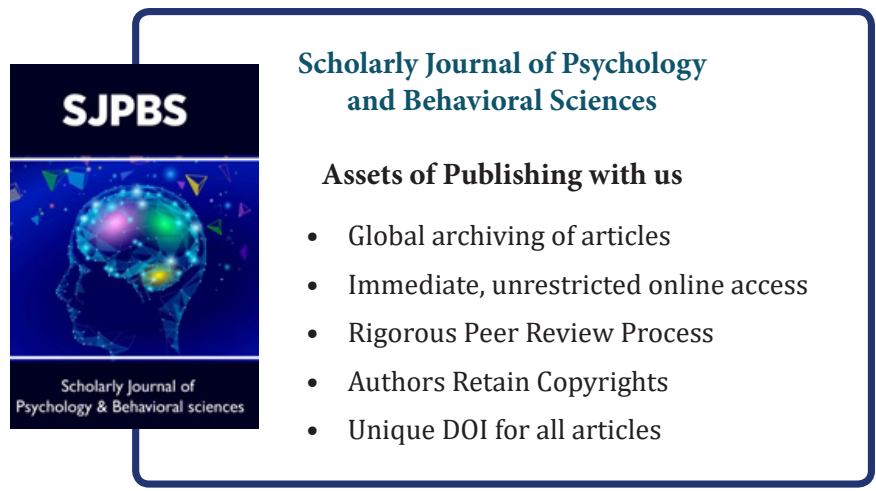

